(C)2009 IEEE. Personal use of this material is permitted. However, permission to reprint/republish this material for advertising or promotional purposes or for creating new collective works for resale or redistribution to servers or lists, or to reuse any copyrighted component of this work in other works must be obtained from the IEEE. 


\title{
A Digital Information Ecosystem Structure for Regional Climate Change Assessment and Adaptation
}

\author{
Omar Hussain, Antuaned Perdomo, Tharam Dillon and Elizabeth Chang \\ Digital Ecosystems and Business Intelligence Institute \\ Curtin University of Technology, Perth, Australia \\ e-mail : \{O.Hussain, T.Perdomo, Tharam.Dillon, Elizabeth.Chang\}@cbs.curtin.edu.au
}

\begin{abstract}
Global warming is one of the most important and challenging problems being faced this century, capable of triggering a series of events that will have serious consequences on the different ecosystems on planet Earth. In order to manage such consequences, we need dedicated actions for resilience. In such a scenario, the process of adaptation to climate change plays an important part. This is achieved by first identifying the various events of risks, assessing the level of their impacts, and then developing policies by which they can be mitigated. For effective adaptation, the events of global warming should be identified and assessed on a regional scale. In this paper, we propose a digital information ecosystem architecture for climate change adaptability. The proposed architecture will capture the different regional factors of global warming which can then be linked together to produce effective macro adaptation policies.
\end{abstract}

Index Terms-Global Warming, Climate Change, Macro impacts, Micro Impacts, Multi-system and Adaptation.

\section{INTRODUCTION}

Climate Change has become an important topic of discussion because of its alarming and potentially serious effects on planet Earth. The International Panel on Climate Change (IPCC) defines climate change as the change in the state of climate that can be identified by the changes in the main (and or the variability) which exists for a persistent period of time. One of the reasons for climate change is Global Warming, which is already affecting all the different aspects on which the humans depend for their daily tasks and lifecycle. As the name signifies, Global Warming means the warming of temperatures around the world. This is caused by an increase in the Greenhouse gases and other heatabsorbing gases such as carbon dioxide, methane, nitrous oxide etc., which are trapped in the Earth's atmosphere when it is heated by the sun. The steep rise in the increase of greenhouse gases is caused by the increase in the consumption (burning) of fossil fuels by the inhabitants of the world [1]. According to a report from Jones and Moberg, planet Earth has warmed by $0.7{ }^{\circ} \mathrm{C}$ on average since 1900 [2]; and most of the warming is due to human activities [3].

The effects produced by the warming of the Earth are experienced in different areas and ways in different parts of the world. For example, some parts of the Earth are experiencing droughts and famine, whereas other parts are being flooded due to heavy rains, storms etc. The list of consequences does not stop here. It extends to other ecosystems and multi-systems which are in turn dependent on the previous ones; for example: agriculture, vegetation, health, envi- ronment, living ecology etc., all of which are dependent on the climate for achieving their desirable aims. A multisystem as shown in Figure 1 is a long term dynamic interaction between natural, artificial and abstract systems. A natural system is defined as the earth and universe system without human intervention. The earth system is a dynamic combination of different living and non-living elements. An artificial system is created by humans for their different needs. Abstract system is the combination of natural and artificial system created by humans. If the climate is affected as a result of global warming, then it will have a snowball effect on the different ecosystems and multi-systems that are dependent on it. It may also have an effect on a country's economy and, perhaps, also the world's economy.

From the figures that we have at hand, global warming is already having an effect on all the different factors on which humans depend in the course of undertaking their daily tasks. In order to prevent the effects of global warming from creeping into and disrupting the equilibrium for humans in their day-to-day life scenarios, adaptation is very important. To facilitate this adaptation, steps can be taken by which the different types of risks are identified, assessed and managed. But it is important to note that the process of adaptation is a sequential and iterative one. In this paper, we propose a digital ecosystem information architecture which can be utilized for climate change adaptation. The proposed architecture will be utilized to consider and model various possible scenarios and impacts from the different factors affecting climate change. The paper is organized as follows. In Section 2, we discuss some of the causes and effects of global warming. In Section 3, we classify the impacts of climate change according to two broad categories. In Section 4, we propose the digital ecosystem information architecture for adapting to climate change and then discuss the importance of such architecture to regional and micro adaptation. Finally in Section 5, we conclude the paper.

\section{Causes And Effects Of Global Warming}

Climate is the result of long-term dynamic interactions between multi-systems including the dynamic process of the Earth's systems and external forces, such as the variation in the sun's radiation, greenhouse gas (GHG) concentrations, geographic location and anthropogenic activities. Moreover, there are several other factors such as ocean temperatures, winds and volcanoes which could shift the patterns of climate in a short period of time. Unfortunately, the energy 


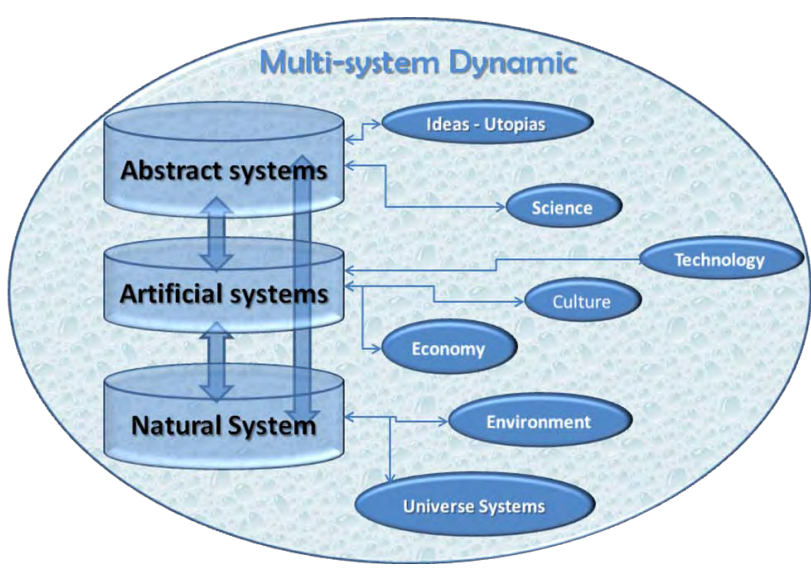

Fig 1. Description of a Multi-system

balance of the climate system of the Earth has been altered, which has resulted in Global Warming. The main stressor for this is the increase in gas emissions to atmospheric concentrations. Carbon dioxide $\left(\mathrm{CO}_{2}\right)$ is emitted by the use of fossil fuels for energy supply, transport, and industries. Methane $\left(\mathrm{CH}_{4}\right)$ is produced by decaying degradable matter, e.g. in landfill sites and from livestock. Then there are nitrous oxides $\left(\mathrm{N}_{2} \mathrm{O}\right)$ from fertilisers, industrial processes, and fossil fuel burning; and a group of other gases, such as perfluoromethane $\left(\mathrm{CF}_{4}\right)$ and perfluoroethane $\left(\mathrm{C}_{2} \mathrm{~F}_{6}\right)$ used in aluminium production, and sulphur hexafluoride $\left(\mathrm{SF}_{6}\right)$ from dielectric fluids [4]. Other gases, such as carbon monoxide (CO) and nitrogen oxides (NOX) have indirect effects on greenhouse warming through various chemical reactions [5]. The atmospheric concentration of GHG due to emissions exceeds Earth's natural removal process. Over the last century, the GHG emissions has increased by approximately $80 \%$ [5], causing a steep rise in global warming and consequently creating uncertainty over the future of the natural ecosystems and humans. The weight of the different gases in the variation of GHG and the increase in temperatures change depending on the "Global Warming Potential" (GWP) [6]. The main gas polluters are carbon dioxide 1 (GWP), methane 23 (GWP), nitrous oxide 296 (GWP), hydroflurocarbons 12 to 12000 (GWP), perfluorocarbons 5000 to 12000 (GWP) and sulphur hexafluoride 22200 (GWP). Even if carbon dioxide is equal to 1 (GWP), it remains the most important gas because of the quantities in which it is being emitted.

The scientific community's main concern about Global Warming is not the fact that the temperature of the Earth has risen since pre-industrial times (from approximately 100 years), but of the fact that the global GHG emission has increased by $70 \%$ from 1970 to 2004 [7]; exceeding the expected average patterns. About $80 \%$ of the world's energy is currently derived from burning fossils fuels. Not having a clear international policy of trade and the reductions of emissions does not help the situation either. It is projected that the continuing addition of carbon dioxide and other greenhouse gasses to the atmosphere will increase the global temperature of 1.4 to $5.8^{\circ} \mathrm{C}$ greater than what it was in 1900 [8]. Apart from the emission of such gasses, the Earth observatory shows that the Sun's energy has fluctuated in the last decade and is most likely to have a measurable impact on the Earth's atmosphere. Researchers believe the rise in sunspots and faculae since the late seventeenth century may be responsible for as much as half of the 0.7 degrees of global warming over the last 110 years [9]. The consequences of the increase of temperatures due to such activities are irreversible, and their impacts on the different ecosystems and human settlements have to be studied in detail. A multi-system response to climate change could trigger non-linear responses and have secondary impacts on different ecosystems. The best option for the present is to have a process of adaptation, by which the risk factors and impacts of climate change can be determined and then policies can be developed by which they are managed at an acceptable level. The process of adaptation should be such that it takes into consideration the effective development of social, economic and scientific dynamics. In the next section, we will discuss the different types of impacts of climate change and classify them into two broad categories.

\section{IMPACTS OF Climate CHANGE}

Even if the descriptions of the effects of climate change are consistent across the world, its impact varies according to the geographical location and different factors in question. The effects and impacts of climate change vary according to the different types of ecosystems that are present on planet Earth. Further, the effect and magnitude of such impacts also vary across the different continents and countries around the world, depending upon their location. A report from IPCC released in April 2007 studies the effects of climate change on the different continents of the world [10]. From the report, it can be seen that each continent is going to be affected in a different way depending upon its location and exposure to the different aspects or factors of climate change. For example, Australia and New Zealand are going to experience an increase in drought and fire, whereas Asia and North America are expected to experience an increase in flooding and winter flooding respectively. Furthermore, the increase in the rate of impact also varies across the different continents of the world. For example, the effects of climate change in North America are far more abrupt than those currently being experienced in Asia. Even within one continent, the effects of climate change are not the same throughout. A report from one UN climate change conference states that South East Asia is going to be affected the most with an expected increase in flooding, whereas by comparison, Japan and China may not be affected as much. So the effect of climate change is not constant throughout the world, but varies according to the location of the specific country under discussion. From such an analysis, the impacts of global warming can broadly be divided into two main categories, namely:

1. Global Impacts, and

2. Regional Impacts.

Global impacts represent the different ways by which the 
planet Earth and its different ecosystems will be affected as a result of climate change. These types of impacts can also be termed Macro effects as they give the broader or bigger picture of impact throughout the world. But as discussed earlier, the magnitude of impacts from the different identified factors may not be the same and constant throughout the world. Similarly, the process of adaptation that needs to be carried out for combating climate change should be done according to the specific region in question; and the specific impacts that climate change might have on it should be ascertained. Such specific impacts of climate change are termed regional impacts of climate change. The regional impacts can further be broken down into different impacts according to a sector which are termed Micro effects of climate change. The micro level impacts take into consideration the specific regions of a country, and study the effect on different multi-systems on a minor scale. In this paper, we use the terms regional and macro effects interchangeably to represent the regional effects of climate change. The process of adaptation that needs to be carried out should be according to the specific impacts by which a particular region is affected, due to climate change. As a result, it should be a bottom up approach, which addresses first the local or regional impacts that are specific to a region. This will eventually lead to solving the global impacts of climate change. In the next sub-sections, we will discuss the global and regional impacts of climate change and then narrow down our discussion to identify the local or regional impacts of climate change on Australia.

\section{A. Global Impacts of Climate Change}

Some of the broad macro effects of climate change are: 1. Increase in atmospheric temperatures.

2. Increase in sea levels.

3. Global loss of biodiversity and ecosystems.

4. Coastal areas erosion.

5. Detriment of food supplies and security.

6. Increase in Health problems.

While the impacts of the macro effects of climate change have been studied in quite some detail by various considerable respected international bodies, the real effects of these can be comprehensively understood only by assessing them on a regional or micro scale. In other words, the accurate macro impacts of climate change on the planet Earth can be determined only when each of the abovementioned factors is studied at the micro level. Whilst considerable works in the form of careful scientific simulations have been done which investigate the macro effects [11], less work has been done on determining the regional impacts. Because of this, there is a lack of understanding of these regional impacts by policy makers and managers of various local and regional areas. In the next sub-section, we will discuss the regional impacts of climate change.

\section{B. Regional Impacts of Climate Change}

As discussed earlier, the impacts of climate change are not constant throughout the world, but vary according to the specific geographic locations. So, in order to understand their effects properly, the key areas that need to be studied at the micro level are:

1. Water: Water is an essential resource for all sectors and regions. According to the IPCC report, the current knowledge about future impacts by the increase in sea levels associated with ice cap melting, increase in temperatures envisages changes in precipitation, thereby increasing the risk of floods and drought in many areas [12]. The sea level also affects the salinity of ground water, resulting in a decrease in fresh water availability for humans and other different ecosystems. The rises in temperature cause an upheaval in the circulation of the oceans which in turn causes abrupt climate changes and disturbances in the winds, thereby creating natural disaster.

2.Biodiversity: Global losses of biodiversity are irreversible. Factors such as ocean acidification, droughts, floods etc can exceed the limited resilience of the different ecosystems. These factors, when combined with human activities such as land use, urbanization, pollution etc increase the risk to the survival of ecosystems. Approximately $20-$ $30 \%$ of plants and animals are in danger of extinction if the temperature rises by $1.5-2.5^{\circ} \mathrm{C}[13]$.

3. Environment: Erosion of coastal areas very likely, thereby damaging the infrastructure and affecting human settlements in these areas. Developing countries are the most vulnerable to this type of threat.

4. Agriculture: Food security and supplies will be affected by droughts and floods. At lower latitudes, crop productivity in seasonally dry and tropical regions is projected to decrease, which may result in famine. The cost of food will increase because of the scarcity, which in turn will generate more inequity and social conflicts.

5. Health: Health problems may increase due to the increase in the adverse effects of droughts, famines, floods etc.

The equilibrium in each of the above ecosystems or multi-systems has to be maintained on a regional scale, in order for normal forms of life to exist on planet Earth. Hence, it is imperative to consider, assess and manage the regional impacts of climate change so as to have an effective process of adaptation. Policies for managing risks that are not based on preventing or mitigating the hazards and vulnerabilities by regions, could activate a series of interdependent multi-system consequences. Another factor to be considered while developing the policies is that they should be capable of responding to the different types of events that may occur almost simultaneously in various regions. In the next sub-section, we will highlight the micro effects of global warming which are specific to the regional parts of Australia.

\section{Regional or Micro impacts of Climate Change on Aus- tralia}

According to Garnaut's climate change review [14], the impacts of climate change in six key sectors and areas of Australia that make a large contribution to the country's economy are expected to be severe. The report shows that at the end of the century in no-mitigate scenarios, resource- 
based industries and communities, critical infrastructure, human health, ecosystems and biodiversity, geopolitical stability and catastrophic events will affect Australia. Severe weather events in Australia are expected to increase. By considering the recent bush fires in the state of Victoria and floods in New South Wales and Queensland, it is evident that the effects of climate change are already disrupting human settlements and ecosystems; and the Garnaut report states that in some cases it will be near impossible to avoid some levels of climate change impact. Another report by the Australian Greenhouse Office [15] and CSIRO [16] studies in detail the different areas which will be impacted upon as a result of climate change in Australia. It also states the different impacts on 10 different parts of Australia due to climate change by 2030. Some of these worth mentioning are:

- By 2030 the air temperatures in Australia will rise by $1.0^{\circ} \mathrm{C}$, with warming of around $0.7-0.9^{\circ} \mathrm{C}$ in coastal regions and $1-1.2{ }^{\circ} \mathrm{C}$ inland. This will affect livestock, agriculture and plantation due to the consistent warm temperatures and increase in the consumption of energy.

- The solar radiation is expected to increase more in the southern part of Australia compared to the other regions. This will act as a trigger to produce more droughts, especially in the south-west and also increase the risks of experiencing more bush fires especially in the south eastern part of Australia which is among one of the three most fire-prone areas in the world.

- The rate in the rise of sea levels due to global warming has increased and it is now expected to rise by at least $1 \mathrm{~m}$ by the year 2100 . The rise will also be due to a surge in storms and increase in the intensity of wind. This will result in erosion, submerging of the coastal areas and damage and loss of existing infrastructure. The proportions of intense categories tropical cyclones are expected to increase. Precipitation across Australia is also expected to increase.

- Australia is a maritime nation with a vast ocean that generates $8 \%$ of its gross revenue through fishing, tourism, and recreation. Increase in temperatures is expected to increase the acidification of the ocean water that will affect marine life.

It is possible that some large effects of climate change may be difficult to be avoided, but their magnitude can be controlled to a certain extent through adaptation. So, in order to have a detailed and comprehensive process of adaptation, all these factors along with their possible impacts on different ecosystems, have to be investigated in detail by using simulations. Furthermore, the determined analysis and results should be demonstrated by using a portal as it will educate people and increase awareness among them. What we need is to develop a digital ecosystem which can be utilized to capture all the relevant micro factors of climate change and study their level of occurrence. Based on that analysis, the digital ecosystem should be able to determine their effects on different ecosystems and multi-systems by using simulations, and then develop risk mitigation policies according to the specific region in question. In the next section, we will give a brief overview of our proposed digital information infrastructure ecosystem which can be utilized to study the various micro factors of climate change.

\section{MULTiSYSTEM DigitAl ECOSYSTEM ARCHITECTURE FOR CLIMATE ADAPTATION}

One of the main factors to be considered when developing a digital information ecosystem infrastructure for multisystem adaptation is that it should track and visualize the different aspects of impacts on regional areas arising from global warming. This will require effective data capture, gathering, storage, analysis and process of this information in a way that preserves its semantics. However, the information to be collected may be from different diverse sources and at different technical levels, processes and specifications. We classify such information at three levels of abstraction. They are:

1. High level of abstraction: This includes information received from geospatial technology which provides visualization of location through web applications in three technologies systems: GPS (Global Positioning System), GIS (Geographical Information System), and RS (Remote Sensing). This information could include different time scale sequences in different applications.

2. Medium level of abstraction: This is the information obtained from semi-structured data and sectors analysis, historical data such as socio-economic analysis, energy sector, water sector, industry etc.

3. Low level of abstraction: This is the unstructured information (like text, images etc), and social net working monitoring, real time data observations on a micro-system scale or local scale, collection of real-time data using sensors, volunteers, social networks, wikis, blogs etc. This information may include variability in the weathers, rivers, ecosystems, etc.

Once the information has been captured from different sources, the following steps need to be executed:

(a) Storing of the information across different information repositories. An important point to ensure while storing is that the integrity of the information is maintained.

(b) Classifying the relevant information according to the different multi-systems.

(c) Functionalities to simulate and process the information according to the desired outputs.

(d) Effective visualization and representation of information taking into consideration different levels of geographic locations' granularity and the specific factors to be represented depending upon the specific location.

(e) Development of risk mitigation and adaptation techniques for addressing micro effects of climate change, specific to a regional sector.

(f) Aggregation of the different micro effects to compute and simulate the various macro effects of climate change.

(g) Development of risk mitigation and adaptation policies for risk management for macro effects of global warming.

Figure 2 represents the proposed digital ecosystem infrastructure for adaptability to micro effects and in turn macro 


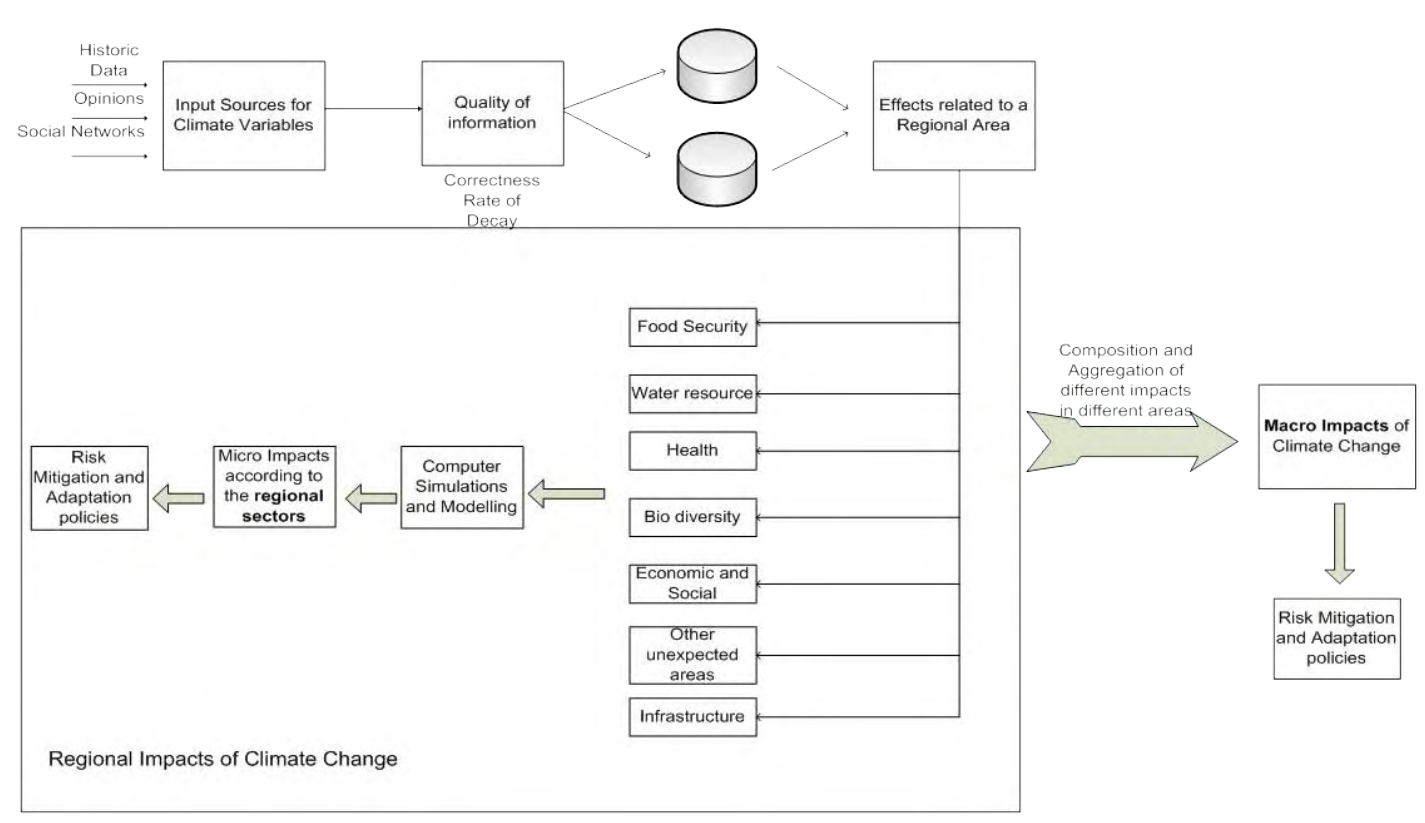

Fig 2. Multisystem Digital Ecosystem Architecture

effects of climate change. The information to be collected can be from a variety of different sources, such as: direct input of opinions by humans, collection of real-time data by using sensors, geographic data, volunteers, social networks, wikis, blogs etc. So it is possible that it may be in different heterogeneous formats like text, images and audio feeds etc and has to be stored in different database architectures like relational, structural, semi structure etc. But before doing so, it is important to check the quality of the information to ensure its correctness. This information forms the source from which the different computations and simulations will be performed, so care has to be taken that its correctness is checked and its integrity and semantics are maintained and preserved. This is done by annotating and structuring the information in an ordered way or according to a pre-defined standard format by which it can be classified easily at a later stage according to each ecosystem and also ensures seamless interoperability of data across different repositories. Simulations help to determine and model the different scenarios and impacts that could be experienced over a given period of time. But while doing so, it may be necessary to aggregate, composite and transform the information according to different information repositories in order to achieve the required output. The integration of information across the different databases relating to different ecosystems should be dynamic in order so as to obtain the required relevant information from different repositories. To determine the consequences, we need to simulate the impacts on different ecosystems due to the identified factors. Factors of vulnerabilities need to be simulated and forecast in different scenarios of occurrence, and their effects be assessed on the current social and infrastructural problems, such as poverty, unequal access to resources, food security, environmental degradation, education, infrastructure and social capital. Having such visualization will help us to develop strategies which such impacts can be mitigated in different ecosys- tems. This will result in 'sustainability' which is defined as preservation of the important ecosystems over time, irrespective of the different scenarios that may occur.

The computed information may need to be accessed by a variety of people from different domains such as environmental scientists, regional managers, decision makers and policy formulators at both regional and national levels. Each of them may require the processed information to be displayed at various granularities of detail and hence, presentation of the information is a vital issue. For easy understanding and better education, it is important that the digital ecosystem have the functionality to represent the computed information at different levels of abstraction. This will help the computed information to be represented according to the end users' requirements, depending upon their level of expertise. To account for the composition of the processed information and services combined with information visualization and animation at distributed sites, it is necessary to have a digital ecosystem that employs service oriented architecture through semantics, ontologies and targeted markup languages. Based on the composited information, risk management policies can be developed and then displayed to the users through various techniques by which adaptation can be carried out in the real world. The determined micro effects and impacts of climate change, when aggregated, will represent the macro impacts of global warming which represent their effects on the real world.

\section{Application of the Digital Ecosystem Architecture for Regional Adaptation}

The proposed digital ecosystem architecture follows a social network approach for identifying, assessing and modelling the impacts as the result of climate change. This is different from having a model that is completely dependent on using historical and real time data. Real time monitoring by using satellites and sensors is an effective approach for 
having a reliable process of risk assessment, but high temporal resolution via satellite to regional areas is often technically difficult and very expensive. As a result, it is very difficult to have an analysis and apply it to all the different locations around the world, especially in developing and remote areas. Although the benefits and advantages of utilizing real time analysis is the best solution if we are to face the threats of climate change, other alternatives must be considered due to financial and infrastructure concerns. This is achieved by the proposed digital ecosystem architecture for climate adaptation that uses different possibilities for inputs like real time data analysis, historical data, blogs, wikis, personal opinions etc.

The proposed digital system architecture can be utilized to develop an "Early Warning System" which can be utilized to mitigate the impacts of climate change on human's settlements and other vulnerabilities. The proposed ecosystem can be utilized to create an easy understanding of the possible future scenarios by using simulations. These can be utilized to develop policies by which the current or future similar events in different locations can be managed and averted beforehand, under the same or approximate conditions. The importance of early reaction to and evasion of an event can not only mitigate the impact on one region, but can also be beneficial across regions in other ecosystems. However, in order for the developed policies to be successful and easily adapted, it is necessary for the ecosystem to represent the impacts and the necessary adaptations in an easily comprehensible manner.

\section{CONCLUSION}

Global warming is as an important area of concern in the current world. One of the triggering effects that has resulted from it is the change in the climate of planet Earth. Climate change in turn will have a series of consequences on the different ecosystems that are dependent on it. In this paper, we examined the various impacts of climate change on the different ecosystems of life on planet Earth and classified them into two broad categories, namely global (or macro) and regional (or micro) impacts. We studied each category of impact in detail and then highlighted the importance of considering the micro effects while developing the policies for adaptation to climate change. We then proposed a digital ecosystem based information architecture for capturing, storing, monitoring, simulating the various factors as the result of climate change and then determining their impacts on the different multi-systems or on different ecosystems. The proposed architecture can be utilized by different peoples to represent the information about different ecosystems and according to different levels of granularity. In our future work, we will concentrate on utilizing the proposed digital information ecosystem for developing adaptation strategies in order to ensure water sustainability in regional Australia.
[2] P.D. Jones and A. Moberg, "Hemispheric and large-scale surface air temperature variations: An extensive revision and an update to 2001," Journal of Climate, vol. 16, pp. 206-223, 2003.

[3] J. T. Houghton, Y. Ding, D. J. Griggs, M. Noguer, P. J. Van Der Linden and D. Xioaosu, "Summary for Policymakers: Climate Change 2001: The Scientific Basis, Contribution of Working Group I to the Third Assessment Report of the Intergovernmental Panel on Climate Change," 2001.

[4] Greenhouse Gas Division Environment Canada, "National Inventory Report 1990-2005: Greenhouse Gas Sources and Sinks in Canada,," Ottawa 2007.

[5] Intergovernmental Panel on Climate Change (IPCC), "Climate Change 2001: Synthesis Report," 2001.

[6] Economic Affairs Committee, "The Economics of Climate Change, Volume 1," 2005.

[7] Intergovernmental Panel on Climate Change, "Summary for Policymakers," 2007.

[8] Intergovernmental Panel on Climate Change, "Summary for Policymakers. In: Climate Change 2007: The Physical Science Basis. Contribution of Working Group I to the Fourth Assessment Report of the Intergovernmental Panel on Climate Change," New York 2007.

[9] Ker Than, "Sun Blamed for Warming of Earth and Other Worlds,"

[10] Intergovernmental Panel on Climate Change, "Climate Change 2007: Synthesis Report Summary for Policymakers," 2007.

[11] Modeling Effects of Climate Change on Wildlife Habitats and Species in Alaska, http://www.fs.fed.us/pnw/research/climatechange/project-summaries/AKHabitatCC.pdf.

[12] Intergovernmental Panel on Climate Change, "Climate Change and Water," 2008.

[13] P. I. W. Group, "Climate Change in Australia," 2007.

[14] Ross Garnaut, "The Garnaut Climate Change Review," 2008.

[15] Australian Greenhouse Office, "Climate Change: An Australian Guide to the Science and Potential Impacts," 2003.

[16] CSIRO, "Climate change scenarios for initial assessment for risk in accordance with risk management guidance," 2006.

\section{REFERENCES}

[1] Nature, "Fossil fuels: the evolution of human culture," 2003. 